\title{
LUGARES DE EXCLUSIVIDAD Y EXCLUSIÓN: DE LA CASA AL BARRIO Y DE LA CIUDAD AL ISLOTE
}

\author{
Charikleia Pantelidou \\ International Hellenic University, Department of Interior Architecture, Serres, Greece, \\ lilapante@gmail.com
}

\section{RESUMEN}

En las últimas décadas nos enfrentamos a una transformación urbana basada en la exclusividad. Los barrios cerrados se encuentran en todo el mundo en un intento de las clases medias de protegerse frente al otro mediante la práctica de métodos de injusticia espacial. Sin embargo, lo que distingue el caso de los barrios cerrados de otros métodos de exclusión urbana es el hecho de que la exclusión del «otro» es coherente con la restricción de lo «normal» mientras que al mismo tiempo los conceptos de interior y exterior se reinterpretan y redefinen, en referencia a la espacialidad. En este contexto, la ciudad contemporánea adquiere nuevas formas y significados, alejándose de la organización espacial contemporánea y las correlaciones habituales de formaciones espaciales y sociales. Surgen las preguntas: ¿Cuáles son las cualidades espaciales y sociales de los barrios cerrados? y ¿cómo el fenómeno de los barrios cerrados transforma el espacio urbano moderno. A través de la elaboración teórica del tema y el análisis del contenido de una novela de literatura contemporánea intentamos conocer las transformaciones e inversiones que se producen en el paisaje urbano en la realidad contemporánea de la exclusión espacio-social.

\section{Palabras clave: vivienda, interioridad, lími- tes, gated communities, diversidad}


methods of spatial injustice. What is distinct though at the case of gated neighbourhoods is the fact that the exclusion of the 'other' is combined with the (self-)restriction of the 'normal', while at the same time the concepts of interior and exterior, in reference to spatiality, are being reinterpreted and redefined. In this context the contemporary city appears in new forms that deviate from the Modern spatial normality and the usual correspondences of spatial and social structures. The following questions are explored here: What are the spatial and social qualities of the gated neighbourhoods? And, how has the socio-spatial phenomenon of gated neighbourhoods transformed the contemporary urban space? Based on theory and content analysis of a novel, this paper aims to investigate the ways that the urban landscape is being reversed through the contemporary urban reality of the socio-spatial exclusions.

\section{Keywords: housing, interiority, boundaries,} gated communities, otherness

\section{RESUMO}

Nas últimas décadas, enfrentamos uma transformação urbana baseada na exclusividade. Bairros e comunidades fechadas, uma tentativa das classes médias de se protegerem do outro, por meio da prática de métodos de injustiça espacial, são encontradas em todo o mundo. No entanto, o que distingue o caso dos condomínios fechados de outros métodos de exclusão urbana é o fato de que a exclusão do "outro" é consistente com a restrição do "normal", enquanto que, ao mesmo tempo, em referência à espacialidade, os conceitos de interior e exterior são reinterpretados e redefinidos. Nesse contexto, a cidade contemporânea adquire novas formas e significados, distanciando-se da organização espacial moderna e das correspondências usuais de estruturas sócio espaciais. As perguntas surgem: Quais são as qualidades espaciais e sociais dos condomínios fechados? Como o fenômeno espaço-social das comunidades fechadas transforma o espaço urbano moderno? Baseado na teoria e na análise de conteúdo de um romance de literatura contemporânea, este artigo investiga transformações e inversões que ocorrem na paisagem urbana por meio da realidade urbana contemporânea de exclusões sócio espaciais.

\section{Palavras-chave: residência, interioridade, limites, condomínios fechados (gated com- munities), alteridade}

\section{INTRODUCCIÓN}

Hoy en día, la expansión de la diversidad social, en cuanto a las formas y la intensidad que asume, se traduce en la transformación de las ciudades, por un lado, con la aparición de nuevos tipos espaciales y, por otro lado, con la reestructuración de la organización urbana y la redefinición de las relaciones que conectan formas, significados y usos urbanos. Por diferencia con los espacios tradicionales de exclusión y control y los nuevos "de hospitalidad", en las últimas décadas han aparecido con especial fuerza las instalaciones cerradas, que conciernen a todos los usos urbanos y especialmente a la vivienda. En estas instalaciones se aíslan las clases medias para evitar una diversidad indeseada, molesta, amenazante y peligrosa que tiene la convivencia en la ciudad. El ingreso a 
estas áreas es exclusivo y controlado, mientras sus altos muros están destinados a mantener fuera la diversidad, el crimen, el miedo y la degradación, pero también a preservar los precios de la tierra, el funcionamiento del mercado inmobiliario, el privilegio económico y la calidad de vida de sus habitantes ante la amenaza existente por la proximidad a «otras» áreas.

La práctica de separar los grupos sociales en el espacio urbano y la exclusión de los peligrosos no es una innovación de la época sino un método político de fortalecimiento y mantenimiento de la estructura social, que en tiempos de crisis se intensifica y toma formas extremas. Lo extraño de la organización urbana moderna es que la exclusión de los "otros" se combina con el encierro de los "ciudadanos" y esto ocurre a través del establecimiento de una nueva morfología del espacio urbano, pero también a través de una re-comprensión de su propio significado. En este trabajo se investiga el fenómeno de las comunidades cerradas a nivel de barrio y las espacialidades que se establecen en la nueva composición del espacio urbano, al mismo tiempo que busca las formas en las que se entrelaza en el contexto del tratado contemporáneo. En este sentido, la idea principal que trata el trabajo se refiere a la importancia que tiene la transferencia de la "cerradura" desde la vivienda al barrio para la forma y el significado del espacio urbano contemporáneo. En este contexto, además de la investigación bibliográfica crítica del fenómeno, se estudió un barrio cerrado, como se describe en la novela Tortilla Curtain de T. C. Boyle a la que se aplicó el análisis de contenido. El objetivo del trabajo es contribuir a un enfoque interdisciplinario de la ciudad contemporánea y crítica anatómica de los fenómenos que se alejan, pero donde al mis- mo tiempo se hace imprescindible la exigencia de un ethos espacial de derecho.

\section{TEORÍA EL CONTEXTO}

Los barrios cerrados se establecieron como un tipo residencial a partir de la década de los 70, mientras que en las décadas siguientes ya se habían extendido a escala global. Su colocación en el tiempo traiciona las condiciones particulares que acompañaron su aparición. La magnitud de la brecha social y la desigualdad, la exacerbación de la diversidad en relación con la inmigración generalizada, la retirada del Estado-nación en el contexto de la globalización y la transferencia de responsabilidades tanto a los organismos supranacionales como a los individuos describen el término de vecindario del barrio cerrado (Sassen 2010; Bauman 1997). El cambio simultáneo de interés en la gestión de lo parcial y lo superficial, en lugar de buscar relaciones causales en el contexto de una planificación global, contribuyó a la intensidad del fenómeno, atribuyendo a la planificación urbana el poder de terapeuta social.

\section{MIEDO, PRIVACIDAD, PUERTAS}

Los límites materiales de los barrios cerrados, la puerta, la valla, marcan la prohibición de entrada a los no residentes. La forma de los límites sube hasta muros altos y compactos de gran altura y una caseta con un guarda armado (Grant y Mittelsteadt 2004). En una visión general de los límites, el vallado separa un espacio de otro, en cambio la entrada es la ruptura del vallado y la eliminación de la separación y 
en este sentido, constituye un límite negativo. En las gated communities, donde el vallado existe en su plena función, pero la entrada es controlada y selectiva (Fig. 1) y, por lo tanto, adulterada en cuanto a su función natural -es decir, la eliminación de la separación-, los límites tienden a su expresión absoluta como elementos materiales de separación.

No sería exagerado decir que el tríptico de peligro-miedo-seguridad aparece en el debate actual sobre las comunidades cerradas como la quinta esencia del fenómeno. El poder normativo del miedo se extiende a la orientación moderna de la planificación urbana y a la formación de una corriente que ya se conceptualiza como planificación defensiva. La forma y función defensiva de las gated communities consiste en controlar el acceso al barrio mediante la instalación de vallas, así como en ejercer la supervisión mediante la implementación de sistemas de control en la entrada e interior del asentamiento (Fig. 2, 3). El control de lo diferente se consigue, por un lado, a través de la disminución de la calidad del barrio público y, por otro lado, a través de la intensidad de privacidad e introversión. Sin embargo, aparece la paradoja de que el deseo de puertas de los habitantes no se refleja en una eficiencia real de las puertas, lo que indica la búsqueda de alguna función simbólico-ideológica.

Los límites en el espacio pueden tener variedad de funciones y significados, distinguir y excluir o unir, provocar la comunicación o la coexistencia neutral paralela. Con el tiempo, la comunicación diaria entre las personas cambia el habitus y transforma los significados del espacio (Petropoulou 2020).

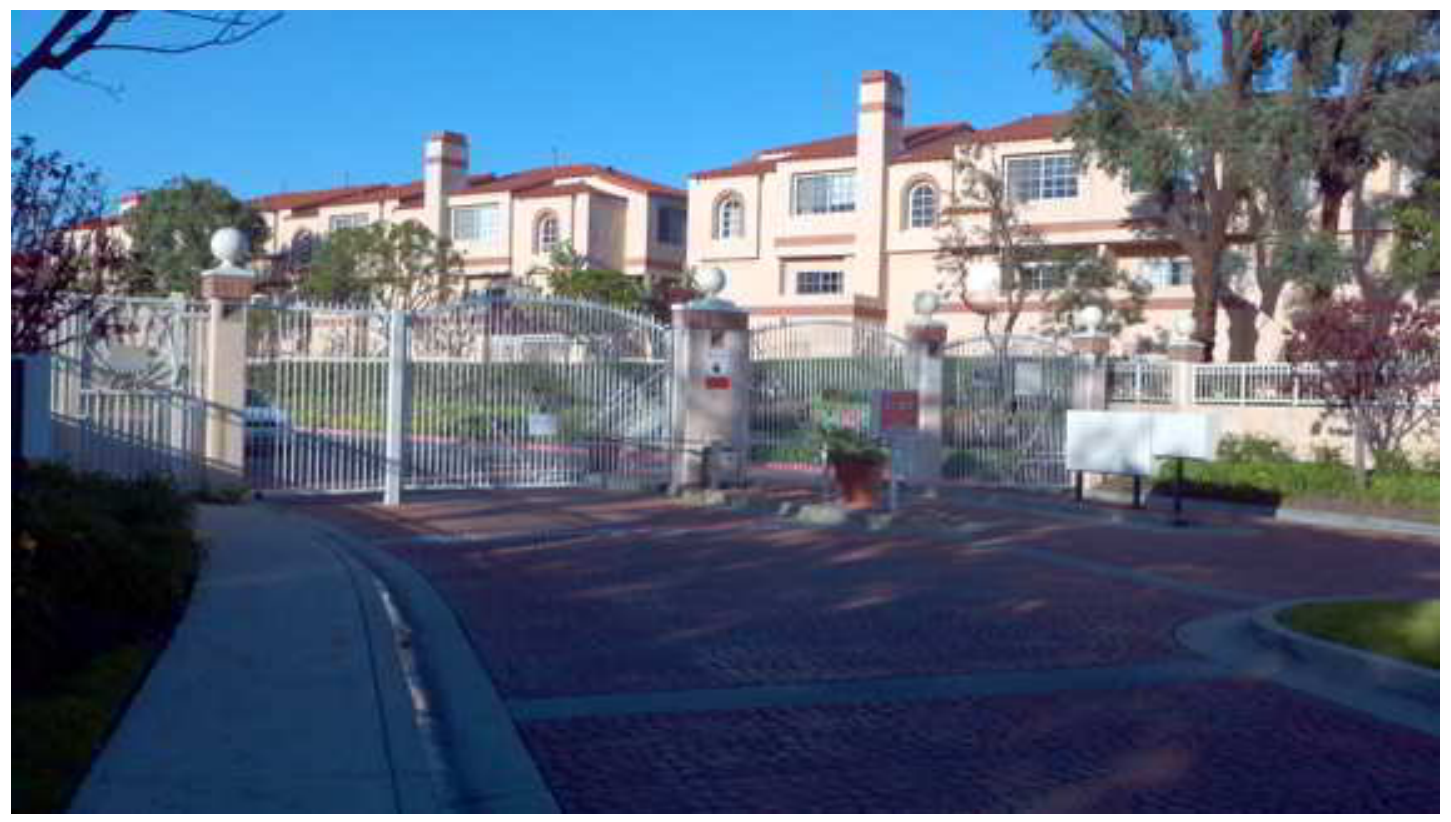

Fig. 1 Gated community en California. De izquierda a derecha: puerta peatonal, salida de vehículos, entrada de residentes, entrada de visitantes. Fuente: el autor. 


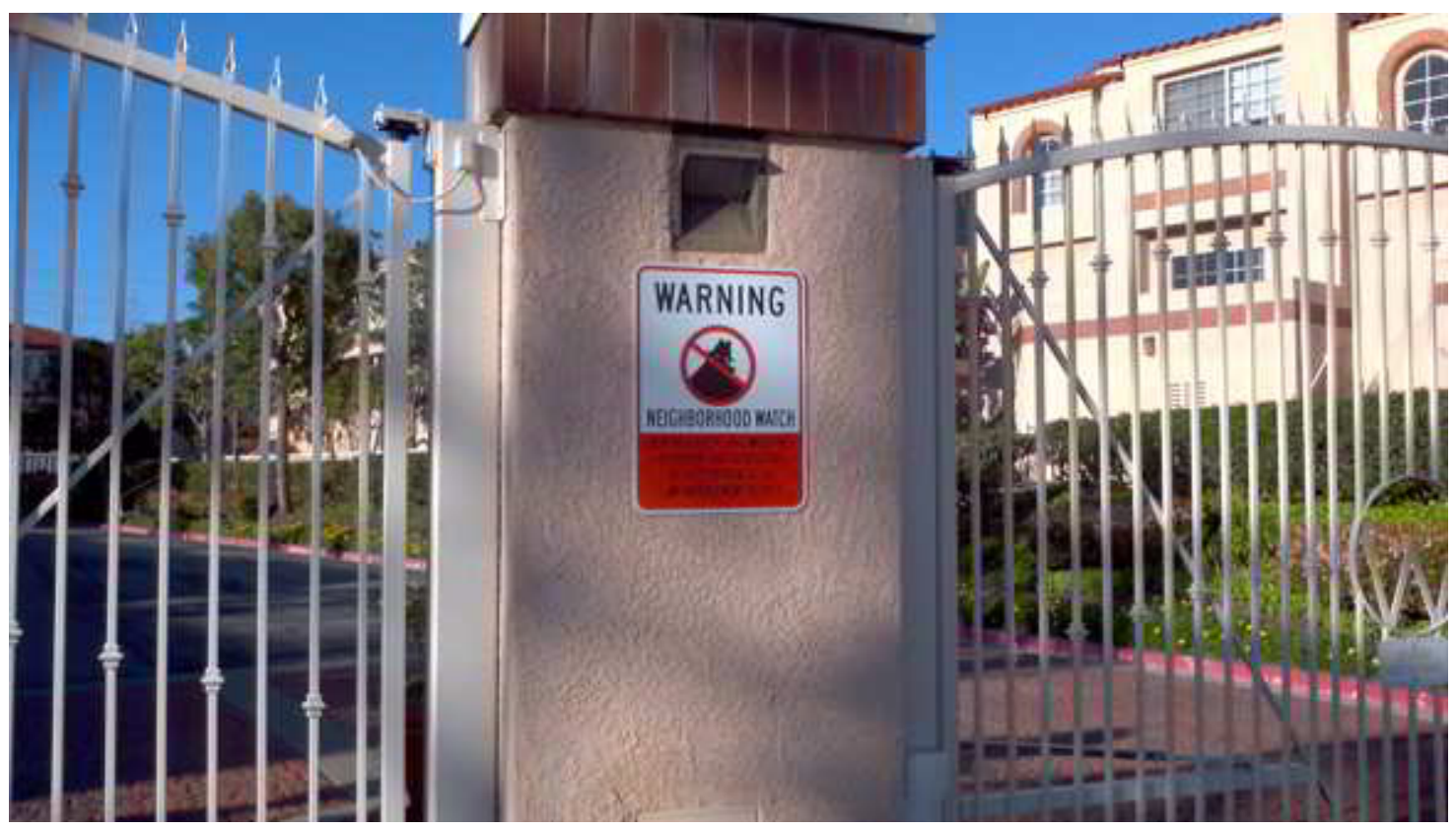

Fig. 2, 3 Gated community en California. Prohibiciones de entrada y advertencias de vigilancia dentro de la comunidad. Fuente: el autor.

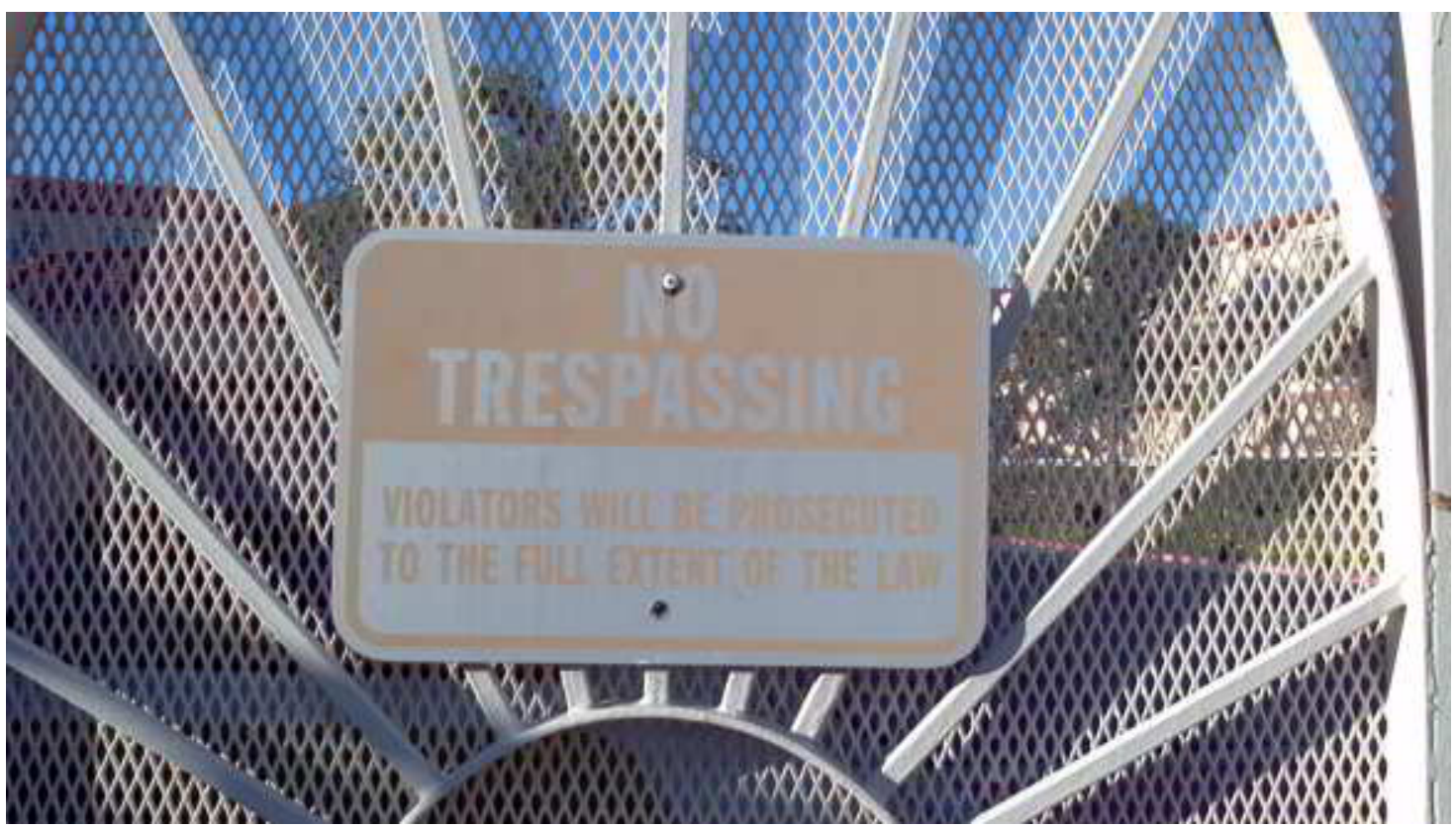


Así, los límites en sí mismos son un término neutral y no significan nada, adquieren significado de las relaciones de las partes de ambos lados y, dependiendo de ellos, pueden significar cualquier cosa (Marcuse 1995). Además, la posición del límite constituye un acto político, ya que el límite actúa como condición para un proceso de correlación entre las partes, aunque sin determinar el tipo de relación (Blakeky y Snyder 1997). En el caso de los límites exclusivos lo que ocurre es la pertenencia, el, ser miembro, alguien que tiene que estar dentro y alguien fuera.

Para Žižek (1989), la fantasía ideológica, la creación de una ilusión, apoya el cierre de la brecha antagónica, la repulsión de la imposibilidad inherente de integración de la sociedad. Los habitantes de las ciudades contemporáneas no tienen el espejismo de que sus puertas proporcionan seguridad (Low 2004), pero siguen confiando en ellas en la práctica, porque pasan por alto que su práctica diaria, su cerramiento dentro de los límites, está estructurado por una fantasía ideológica, un fetichismo de puertas. Sin embargo, señalando al otro como la causa de la segregación social y aislando al externo como un medio de aniquilación, la paz del vecindario adquiere un significado adicional. Consideradas por definición a salvo de la delincuencia, las gated communities en un mundo inseguro y violento mantienen o aumentan su valor de mercado, lo que incita aún más al asentamiento de poblaciones de altos ingresos en la zona (Le Goix 2006). Además, el espacio panóptico de los barrios cerrados supervisado por cámaras, sistemas de vigilancia interna y patrullas, responde a la búsqueda de homogeneidad y coherencia e identidad de los residentes que son adquiridas con su instalación e integración en la comunidad. A través de métodos de exclusión y homogeneización, se destaca la formación de un espacio post-social de islotes exclusivos autónomos para la normalización de lo diferente y el sellado de la brecha.

\section{COMUNIDAD Y ESPACIO COLECTIVO}

Un segundo eje central de análisis de los barrios cerrados es el concepto de comunidad. Es un hecho que el espacio tiene la habilidad de influir en las relaciones del campo social, pero esta habilidad no es independiente de la realidad social más amplia (Tsoukala 2010). En el contexto de un enfoque psicológico de los barrios vecindarios cerrados, la comunidad se identifica con el concepto de sentimiento de comunidad, el cual en la realidad de los barrios cerrados parece limitarse a cualidades de exclusividad (Wilson-Doenges 2000). Además, las gated communities expresan una tendencia general a huir de la urbanización, a deshacerse de los sufrimientos que parecen ser idénticos a los de la ciudad. Esta incriminación de lo urbano revela una nostalgia de lo no urbano como forma pasada de vida.

Sin embargo, las propias gated communities se limitan a la aplicación de alguna forma externa correspondiente a lo no urbano, lejos de abandonar la urbanidad. Esta interpretación de la ruptura posmoderna de forma-contenido, con la reintroducción de la idea nostálgica de la comunidad preurbana en los barrios cerrados, fracasa porque el esquema está desvinculado de la forma y contenido en el espacio y hace que la nueva estructura sea históricamente ineficaz. Además, un espacio narcisista que refleja el grado de individualismo y exclusividad (Kondylis 1991) parece 
constituirse en la forma autorreferencial de las gated communities: el recinto material del barrio cerrado se convierte en un proceso de autorrealización, completación de la identidad rota del sujeto posmoderno, intensificando las cualidades privadas del barrio.

Sin embargo, la dimensión comunitaria de los barrios cerrados también tiene dimensiones políticas. El sistema de administración privado aplicado a las gated communities es parte de la devolución neoliberal de responsabilidades por parte del Estado a los organismos públicos locales y a diversos organismos privados (McKenzie 1994). La organización del consumo colectivo, así como la gestión de funcionamiento del asentamiento son responsabilidades, cada vez, del respectivo esquema administrativo de los barrios cerrados. En la medida que esta forma constituye un método contemporáneo de organización de social, el barrio cerrado forma una unidad estructural espacio-política en la red de las relaciones de poder globalizadas. Además, en la medida en que el barrio cerrado forma una comunidad, asume un papel de político activo más allá de su visión como una agrupación espacial de múltiples unidades de viviendas. De hecho, en el paisaje posmoderno de la micro-política, la división de la esfera pública en territorios micro-colectivos, la plétora de movimientos sociales y acciones donde el carácter progresista o conservador de las reivindicaciones individuales es indiferente, las gated communities constituyen un movimiento social más, el cual esta vez anima a la clase media a la preservación de su privilegio económico.

\section{CASO DE ESTUDIO}

\section{EL TRASFONDO DE LA NOVELA}

La novela que se estudió en el contexto de este trabajo se refiere a los Estados Unidos de América de los noventa en un contexto de intensa migración desde América Latina y los países de Asia y África, así como la ampliación del libre mercado y la brecha social. Esta condición comenzó a gestarse con el aumento de la movilidad de capital, la marginación de la mano de obra y el retroceso del estado de bienestar a partir de la década de los 70, y aumentó aún más las desigualdades existentes en la distribución espacial de bienes, así como en los valores de la tierra dejando a un gran porcentaje de la población sin hogar (Harvey 1992).

La historia de Delaney y Cándido se desarrolla en California, Estados Unidos, en el cañón Topanga al oeste de los Ángeles. La gated community de Arroyo Blanco, está construida al oeste de la avenida del cañón, en el corazón del paisaje natural que se extiende alrededor del cañón y cerca de ciudades y áreas urbanas como Canoga, el asentamiento de Woodland Hills, de Calabasas, de Thoysand Oaks, de Monte Nido. Al este de la avenida del cañón está el Parque Estatal Topanga, donde viven escondidos los migrantes ilegales, Cándido y su mujer América, así como la oficina de Correos en cuyo aparcamiento se reúnen los obreros. Toda la historia trata del enfrentamiento de los dos mundos y el intento de unos por sobrevivir y de los otros por preservar los logros.

La principal distinción espacio-social representada en la novela es la del lugar de residencia entre ciudadanos y no ciudadanos. La primera categoría incluye empresarios, ejecutivos y científicos estadounidenses blancos 
de clase media, cuyas casas están ubicadas en comunidades privadas en las afueras de Los Ángeles. A la categoría de ciudadanos también pertenece la pequeña burguesía americana y los migrantes legales que ya viven en la zona más amplia del cañón Topanga amenazando los valores de la tierra y los nuevos mercados inmobiliarios. A la segunda categoría pertenecen los trabajadores migrantes ilegales de color que viven en un estado de pobreza absoluta y miseria inhumana y están ubicados en el espacio natural del cañón que proporciona vivienda a todo organismo vivo fuera del contexto civilizado urbano.

La comunidad de Arroyo Blanco aparece inicialmente como un asentamiento privado abierto con residencias privadas cercadas. Sin embargo, para hacer frente al peligro natural $y$ al provocado por el hombre, luego se transforma en una comunidad cerrada con la instalación de puertas de control de entrada y un puesto de control en la entrada principal del asentamiento. Finalmente, para mayor seguridad se completa como barrio cerrado con la construcción de un muro de hormigón perimetral en sus límites con el entorno, de 2,10 metros de altura. En el curso de este desarrollo, las medidas de seguridad técnico-espaciales se intensifican para cada una de las residencias privadas por separado.

La comunidad Arroyo Blanco se encuentra en el límite natural y urbano limitando, por un lado, con el auténtico paisaje natural y, por otro lado, con las zonas urbanizadas vecinas. Incluye alrededor de 250 casas, a cada una de las cuales le corresponden una extensión de 1,5 hectáreas, áreas comunes para deportes, circulación y verde, así como un centro comunitario ubicado "en la cima del cerro que domina arri- ba" de la vía principal del cañón y la calle principal del asentamiento. El espacio se distingue por una estricta uniformidad y la organización de las casas es similar con pequeñas diferencias en los planos. Además de su entrada principal, el asentamiento cuenta con otros accesos, que en el recorrido quedan bloqueados por el muro perimetral. El asentamiento tiene carácter político y está autogobernado por un comité de habitantes, mientras que se convocan asambleas generales y extraordinarias donde las decisiones se toman por votación, con escasa participación, sin embargo, de los habitantes. El asentamiento está habitado por una población de trabajadores de clase media. Las actitudes de los habitantes son parte de un marco de privatización y consumismo, mientras siguen un modelo de vida ecológico y resaltan la identidad liberal-democrática, mostrando, no obstante, diferencias ideológicas entre ellos: unos insisten en los ideales humanitarios y las libertades individuales y otros, frente a la percepción del peligro y la creencia de la necesidad, dan paso a prácticas de discriminación y exclusión.

\section{EL IDEAL DE ARMONÍA ENTRE LA CIUDAD Y LA NATURALEZA}

La configuración de límites del asentamiento, así como multitud de prácticas cotidianas de los habitantes forman parte de un clima general de huida de los sufrimientos de las personas y recuperación de un estado de vida más natural. Al mismo tiempo, el asentamiento, aunque construido en la naturaleza, alberga una población que pertenece a la clase media contemporánea, así como un modelo de vida por lo demás urbano en términos de hábitos, comodidades y placeres. En este sentido, el asentamiento es una 
forma híbrida de urbano y natural, mientras que al mismo tiempo su carácter de demarcación limitado intensifica cada cualidad y cada carácter dentro de él. De hecho, la demarcación del asentamiento busca protección tanto del entorno exterior natural como urbano intentando, al mismo tiempo, hacer converger a los dos dentro del asentamiento, dentro de sus límites. En otras palabras, a través de la limitación se busca la unión armoniosa de lo urbano y la naturaleza y específicamente la eliminación del peligro urbano a través de su carácter natural, es decir, una eliminación total del peligro mediante una función natural y urbana complementaria. En este sentido, la demarcación del asentamiento aparece como un escape a una realidad imaginaria, mimética, realidad donde se busca la integración del sujeto en su lugar perdido, la reconexión de su ser roto, natural y social. En el mismo sentido, además, se busca la realización imaginaria del colectivo, esta vez sujeto, a través de la innovación del asentamiento cerrado como comunidad.

Evidentemente se trata de la construcción de un objeto nostálgico, ya que esta supuesta recuperación de una parte perdida y la restauración de la existencia de un sujeto completo nunca existió. La nostalgia expresa esa tendencia en la que el presente es reconocido como un valor inferior al pasado y también manifiesta la necesidad de un proceso de relleno de las grietas y huecos de la imperfecta existencia humana y social. En este sentido, el espacio nostálgico de los barrios cerrados es otro aspecto del diseño con transcendencia política, asumiendo el poder regulador en el presente y en la perspectiva del futuro. Uno diría que el deseo nostálgico del espacio "completo" de un asentamiento seguro y cohesionado se refiere a la noción de melancolía y la consecuente creencia impenitente en un objeto desde el principio ausente, no perdido, de cualidades indefinibles que se desean sin ser conocidas, estimulando estados de ánimo ambivalentes (Freud en Butler 1997). Sin embargo, mientras la identificación melancólica con el objeto ausente es la que asegura su posesión eterna, en el caso de las gated communities, el vacío de la armonía perdida se llena por definición con la propia gated community. En efecto, mientras el aislamiento de un melancólico de la ciudad plantearía cierta demanda simbólica de mejora de la ciudad para que su recuperación dentro de ella valiera la pena, la gated community es una opción positiva, se propone como un lugar mejor de la ciudad, una heterotopía que ya existe.

\section{INTERIORIDAD, EXTERIORIDAD}

Además, durante su transformación en barrio cerrado, el asentamiento Arroyo Blanco fortaleció su existencia interna, que ya había mantenido desde su formación inicial como comunidad privada abierta: la comprensión de la amenaza como externa, que ya existía antes que la gated community, había dado al asentamiento original una cualidad de interioridad, a pesar de que el espacio era abierto, sin límites establecidos. Así el asentamiento formaba su-resquebrajada desde afuera-identidad como interioridad en referencia al exterior. Sin embargo, su transformación en barrio cerrado provoca un impacto en su identidad espacio-social: mientras que inicialmente, antes de ser cerrado, sacaba su interioridad interactuando como abierto con el exterior- y la amenaza externa- desde el mo- 
mento en que se cierra y se aísla del exterior, se constituye ya como un interior autorreferencial y pujante, en un proceso de reconstrucción de su resquebrajada identidad: la exterioridad ahora ha sido eliminada, no forma parte y no distorsiona la identidad del asentamiento.

En este sentido, los enfoques para ver el sujeto como componente desde el exterior -desde la sociedad, la lengua, la cultura- están en desacuerdo con el modelo de gated community, donde, por el contrario, las teorías de autonomía parecen más compatibles. En efecto, la transformación material del barrio en una gated community conlleva consigo toda la carga simbólica de las gated communities, reconstruyendo la identidad social de los habitantes que ahora está determinada por el hecho de su residencia en la gated community. La gated community aislada del resto del mundo como interioridad completa se forma como "imagen" en el contexto de nuestra cultura mercantil, como la describe Debord (2002/1970), proporcionando una versión perfecta y completa de la realidad. Desde un punto de vista lacaniano, esta visión narcisista del asentamiento, la exclusión del de fuera y el cierre de la brecha social a través de su cerramiento, es lo que queda de la realidad si se le quita lo Real. De la forma "virtual" de la gated community, está ausente la causa exacta que condujo a esta forma y la cual pertenece y permanece fuera, en la realidad misma como lugar de competencias y conflictos, imperfecciones y desarmonías. La interioridad del asentamiento, en el sentido definido anteriormente, es decir, con la formación de una identidad autorreferencial a través del cerramiento, se refuerza definitivamente a través de la función simbólica de las puertas. Está activo aquí un fetichismo de las puertas, el cual tiene el significado no del registro exitoso de propiedades que el objeto en realidad no tiene, sino una pérdida de memoria relacionada con sus propiedades reales. De hecho, los habitantes se dan cuenta constantemente de que las puertas no les brindan la seguridad codiciada, sin embargo, como si lo olvidaran de inmediato, buscan cada más intensamente medios para fortalecer el cierre de puertas. Este reconocimiento fetichista del objeto de las puertas de una autoalimentada sustancia intensifica su carácter interior de barrio cerrado ya que, mantiene eficientemente la preservación de su identidad espacial a través de procesos exclusivamente internos.

\section{PRIVADO-COLECTIVO-PÚBLICO}

El barrio cerrado conserva en su interior tanto espacios particulares, como son las viviendas con los patios, los jardines y las sendas, como los espacios comunes públicos, como el centro comunitario, las instalaciones deportivas y los estadios, los aparcamientos exteriores y la circulación en el asentamiento. En estos espacios, los habitantes, realizan reuniones y acciones comunes que tienen que ver con los temas del asentamiento mientras que las relaciones sociales personales entre ellos parece que no existen. En este contexto, surge una nueva cualidad en el ámbito del asentamiento cerrado, en el intervalo entre el espacio público y privado, el de lo colectivo, pero tiene algunas peculiaridades y desviaciones de lo que tradicionalmente percibimos como colectivo. El carácter colectivo de la gated community concierne ante todo a la formación comunitaria del asentamiento a través de su establecimiento espacial único y 
mediante la gestión y administración conjunta de sus asuntos internos, es decir, los que conciernen al territorio y los eventos dentro de sus límites espaciales. Además, el cerramiento del asentamiento refuerza tanto el contenido interior como funcional de asentamiento, o sea, su carácter de colectivo espacial. En efecto, es la forma espacial, el espacio cerrado y encerrado, ya que se basa en los principios de cerramiento, exclusividad y privacidad, que acompaña al carácter colectivo del asentamiento. En otras palabras, el carácter colectivo y el espacio cerrado en la gated communities están directamente conectados y se refuerzan mutuamente: el cerramiento del espacio no es una forma externa de la comunidad privada la cual cambia un contenido claramente estructurado. En efecto, el carácter privado de un asentamiento también puede existir sin la instalación de límites materiales que prohíban y controlen el acceso. Pero el fortalecimiento de la privacidad mediante el control del acceso a la zona colectiva del vecindario produce una nueva cualidad, el colectivo privado, que también parece constituir el tema central de la exclusividad y exclusión producida por este tipo espacio-social concreto.

Así, mientras la comunidad privada esté abierta, es un hecho la clara separación de los espacios públicos y privados: por razones de seguridad, las casas privadas se vallan. Sin embargo, cuando el asentamiento se transforma en una gated community, con la instalación de una puerta de control y un muro perimetral, la distinción entre público y privado -dentro y afuera, controlado y descontrolado- en lo que se refiere a las casas y a los espacios comunes empieza a difuminarse y se transfiere ya a la relación del asentamiento con su entorno exterior. Lo que ocurre es un desplazamiento del límite perceptivo entre el espacio que es suyo, controlado y seguro y el que no controlan y es peligroso, desde la casa particular y el espacio del vecindario al espacio del vecindario y el mundo exterior. Sin embargo, esta transferencia tiene más el significado de la intensidad del espacio privado a través de la expansión de sus cualidades en el espacio colectivo del barrio, que el significado de una apertura de la casa privada en la vida colectiva del barrio.

El desplazamiento del límite del espacio privado de la escala de vivienda a la escala de barrio y el consiguiente significado del barrio como vivienda ampliada significa la expansión del carácter privado-interpersonal de la vivienda y en el barrio. En la vivienda urbana moderna, el espacio privado de la casa se considera núcleo de la esfera privada, y como tal, se enfrenta al espacio social del vecindario, la ciudad y el mundo (Lefas 2009). Sin embargo, en el modelo vivo de la gated community, la esfera privada se extiende más allá de su hogar y cubre, sin más confrontación, el vecindario y el asentamiento. En este contexto, la cualidad del interior en el espacio, que tradicionalmente acompaña a la vivienda y se refiere al espacio cerrado por la atmósfera, se extiende ahora a la escala de barrio e indica la cerrazón que el vallado asegura tanto en los edificios como en sus espacios abiertos. Así, el espacio colectivo del barrio cerrado ya, acoge al mismo tiempo cualidades y características del espacio privado y se extiende como espacio colectivo privado, en lugar de como colectivo público. Esta medida define una dimensión adicional del espacio colectivo de la comunidad cerrada: las cualidades espaciales de un lugar que se dirige a la diversidad colectiva de las personas, como el barrio urbano abierto, cambian y determinan ahora 
el nivel interpersonal de los miembros estrictamente identificados de la comunidad cerrada.

\section{REFUGIO Y ESPACIO DOMINANTE}

La transformación del asentamiento de una simple comunidad privada a una comunidad cerrada significó la exclusión de todo el entorno y los peligros que les amenazan fuera del área que describe los límites del asentamiento y, por lo tanto, la creación de un entorno "puro"en su interior. Esta pureza se refiere a una cualidad de realización y cohesión, que puede ofrecer la homogeneidad social de los habitantes y el mantenimiento de un estilo de vida común, mientras que al mismo tiempo promete mantener la posición de los habitantes en las relaciones jerárquicas del campo social competitivo. En este sentido, la espacialidad depurada orientada al barrio cerrado tiene la importancia de establecer un Todo residencial espacio social, inviolable e invulnerable frente a cualquier factor erosivo amenazante, un Todo que se entiende como la restauración-reparación de la realidad externa dañada dentro de los límites específicos del asentamiento. El control de acceso y la pureza que consigue refuerzan el carácter colectivo del asentamiento, pero con un significado específico: en la medida en que lo sella del entorno espacio-social; es decir, en la medida en que constituye la colectividad como cerrada.

Realmente la demarcación del asentamiento significa el control del acceso mediante la instalación de límites materiales, en lugar de simplemente mediante la declaración de carácter privado del asentamiento $\mathrm{y}$, por tanto, a través de la adquisición en el asentamiento de la cualidad de cierre. Entre las gated community y los espacios exteriores de consumo, trabajo u ocio se recomiendan recorridos axiales con puntos de partida y, finalización en la gated community, mientras los espacios que se encuentran en el otro extremo de cada eje no parecen estar conectados entre sí. Además, en términos de continuidad-discontinuidad, la continuidad se entiende solo por la gated community hacia su espacio exterior, que termina definitiva e irreversiblemente en el límite material del barrio cerrado. En este sentido, la gated community parece investida con la cualidad de una peculiar centralidad como el punto desde el cual las acciones parten y se difunden y en él terminan nuevamente de manera controlada o como otra versión del espacio panóptico desde el cual alguien observa y organiza sin ser él mismo accesible, gozando de esta manera de una primacía de dominación frente a los demás.

Desde este punto de vista, la gated community constituye para sus habitantes un refugio privado de las distopías del mundo contemporáneo, cuyas cualidades de cerramiento, de uniformidad espacial y homogeneidad social se solidifican y refuerzan frente a un exterior amenazador. En lo que respecta a la cualidad específica de la cerrazón del espacio, la gated community se articula en los conceptos de privacidad y la seguridad al articularlos entre sí. Además de esta última articulación, la comunidad cerrada y la práctica espacial del cierre de puertas se entrelazan por cuestiones de riqueza y poder, revelando como el otro lado del refugio el establecimiento de un espacio dominante, cuya función particular en la ciudad contemporánea se asocia a su límite exclusivo unilateral en relación con el resto de la ciudad. 
En su participación en las relaciones sociales de dominación/sumisión, la gated community responde con su especial cualidad colectiva, como un espacio colectivo con cualidades de independencia, ampliado desde el límite de lo privado y encogido desde el límite de lo público. Además, como quintaesencia de la privacidad y seguridad del asentamiento se revela a lo largo del texto el límite entre el interior y el exterior, cerrado y abierto, la cerradura, la entrada. Por el contrario, el elemento más importante para la seguridad de la casa improvisada de Cándido en el espacio natural es el techo, que protege del tiempo, no la entrada. Allí el control de acceso pierde su importancia primordial como herramienta para lograr un espacio seguro. El espacio de la casa en la naturaleza se configurará como seguro con la construcción del techo, mientras que cualquier cerradura en la entrada es indiferente. En este sentido, el control de acceso adquiere significado en relación a la seguridad del espacio solo en el ámbito social urbano y la competencia que lo rige, mientras que en la naturaleza como lugar ecuménico se despoja de su contenido.

\section{CUESTIÓN DE SOSTENIBILIDAD}

Para protegerse del peligro de los depredadores, los que amenazan los bienes de los habitantes del asentamiento, la novela propone dos soluciones. Una conduce a la fortificación del asentamiento con la instalación de límites materiales, los cuales evitarán la invasión del peligro dentro de la comunidad. Este punto de vista busca eliminar el peligro exterior y mantener un área limpia del asentamiento. Al final de la historia, los límites exclusivos del asentamiento se destruyen, ya que resultan ineficaces, y el enfoque de cierre de puertas se señala como insostenible, mientras que se establece una ética espacial de armonía en un contexto de reconciliación, perdón y consenso de ambas partes en las condiciones existentes. La otra solución propone una especie de gestión de la situación de guerra como medio para prevenir los ataques de los oponentes y la posible inversión de las relaciones entre las dos partes. El objetivo es suavizar las relaciones conflictivas y la convivencia armónica de los diferentes a través del respeto del adversario y la comprensión de su propia realidad. Sin embargo, en la medida en que esto se implemente en prácticas de mera no provocación, en una especie de convivencia paralela a través de la convivencia consensuada de cada uno en su lugar, se trata aquí también de una forma de exclusión indirecta. Es comprensible que en este enfoque el espacio funcione, de una parte, como un campo de mitigación social y, de otra, como un campo de aceptación de la diferencia social, una aceptación en la que se basa la crisis sobre la viabilidad de este asentamiento urbano.

Esta versión, además, del respeto entre los diferentes en un marco de convivencia paralela, parece ser coherente con los enfoques económicos de sostenibilidad de la organización del espacio urbano en sectores cerrados. El espacio colectivo privado de las gated communities se propone como una solución de sostenibilidad urbana, en el contexto de una teoría de la eficiencia de las ciudades privadas, un conjunto de espacios-club, para las necesidades del desarrollo contemporaneo (Webster 2002): la idea es que el mantenimiento del espacio es económicamente menos perjudicial si cada uno asume el coste de los espacios públicos que utiliza. En esta medida, la colectividad de las gated 
communities es completamente diferente a otros colectivos con cualidades públicas. Permanece, sin embargo, el hecho de que, en el mundo contemporáneo, colectividades de todo tipo prosperan en un contexto de apoyo a los procesos de autorrealización de los individuos o el fortalecimiento de la individualidad y el narcisismo a través de la integración y discriminación por los restantes del conjunto (Kondylis 1991).

\section{CONCLUSIONES}

Las gated communities están cargadas de una contradicción básica: si bien con su estructura interna invocan una moralidad de la armonía, el principio y el punto de partida de su existencia es la desarmonía del mundo, la cual no buscan corregir sino evitar. La condición cósmica descrita en el texto de la novela se basa en un estado generalizado de competencia, en cuyo contexto también tiene sentido la delimitación del espacio como exclusivo. De hecho, aquí la comprensión del espacio está conectada con la visión del individuo, no solo como distinto de la sociedad en su conjunto, sino también como amenazado por ella, respectivamente la correspondencia entre espacio público y privado, donde el primero se percibe bajo la amenaza de este último en lugar de posiblemente como parte integral de él. Además, lo opuesto, conflictivo, esta correlación de lo privado y lo público, lo individual y lo social, finalmente lo de dentro y lo de fuera, resalta el contraste entre el asentamiento independiente, de relleno y acabado y la ciudad pública en ruinas y elíptica. En esta medida, sin embargo, la correlación de adentro y afuera, como se evidencia en la ciudad de las gated communities implica una negación de la aceptación de la brecha radical posmoderna como principio en cuanto a dentro, en el individuo, en lo privado: el barrio cerrado mantiene esta negatividad como una característica solo de afuera, de lo público, de la sociedad, ignorando que se relaciona tanto con el sujeto colectivo como con el individual. En este sentido, la ciudad de las secciones cerradas revela la ilusión de un entorno de armonía urbana, del que se han borrado las huellas sociales de su producción. En la enseñanza madura de Lacan lo Imaginario se acerca a lo Real: debajo del prójimo-semejante, la imagen espejo, acecha al otro inhumano, el otro absoluto de lo Real, la convivencia con el cual no puede ser en lo más mínimo soportable, salvo si el Otro simbólico interviene, a quien ambos deben someterse, para entrar en una relación intersubjetiva (Žižek 2001). Pues bien, ¿en la gated community, los lugares a ambos lados de los límites no constituyen lo imaginario para el otro, la imagen que para los que están fuera de los muros representa el Ego ideal, lo que les gustaría ser a ellos y para los de dentro el aspecto sucio invertido, en el que nunca querrían caer?, $y$ ¿ ¿es exactamente por esa razón que se encerraron dentro? Y, al mismo tiempo, ¿estas partes de ambos lados no encarnan el otro inhumano el uno para el otro, la diferencia radical dentro de cada uno, los "demonios internos" de ambos que como "demonios" se mantienen alejados, los unos expulsados brutalmente y humillados y los otros habiendo sacrificado su propia libertad, y como "internos" que fueron exactamente demonizados el uno dentro del otro como la amenaza real, los unos de la riqueza de los de dentro y los otros como los generadores de la miseria de los de fuera? Y, sin embargo, conviven gracias a la mediación de las puertas, 
las cuales lograron transformar la monstruosa Cosa de ambos en la representación del simbólico otro único, de un sujeto urbano completo.

Los barrios cerrados sugieren un espacio urbano postsocial, una ciudad de exclusividad y exclusiones, la cual adquiere la forma unida de los tramos cerrados y excluye el resto de la zona urbana. En una gama más amplia de técnicas de control en el contexto de una gubernamentalidad espacial que asume la gestión de los habitantes (Foucault 1991), la centralidad de la comunidad cerrada se traduce en un parámetro de gestión del orden social y económico de la ciudad. En esta versión contemporánea de organización urbana es que ahora son los estratos superiores los que están aislados, en lugar de los estratos sociales inferiores los cuales ahora utilizan la ciudad abierta. Específicamente, en la disposición contemporánea del espacio urbano en islotes exclusivos individuales, sucede que el contenido de la ciudad, las funciones y usos que componen la vida urbana, así como los respectivos gobernantes de la ciudad, se trasladan dentro de los límites de los islotes. Se trata del traslado de la ciudad, de la vida urbana y las personas que la operan, desde el espacio urbano abierto de la plaza, las calles y los edificios, desde la ciudad pública como la conocíamos hasta hoy, a las áreas exclusivas controladas que ahora se hace cargo de la realización de lo urbano. En el orden socio espacial introducido por las gated communities, la ciudadanía se combina con la privatización y la abstención de la vida pública. Por el contrario, la naturaleza de lo público concluye en el "otro" marginal.

Respectivamente, el espacio urbano se transforma. Las funciones urbanas se trasladan al islote, en zonas controladas de carácter exclusivo, que ahora dan forma y significado al concepto de ciudad. Los centros comerciales, de trabajo, de ocio, de servicios, de educación y de salud se ubican en espacios cerrados y vallados, en barrios espeluznantes dentro o fuera de la ciudad, donde lo urbano se percibe como relacionado con cualidades de cercanía, demarcación y exclusión. En la ciudad que queda libre, se deja la vida marginada que tradicionalmente estaba asentada en espacios cerrados estigmatizados y en régimen de exclusión de la ciudad. La vida marginal e ilegal del mendigo se desarrolla en el espacio libre de la ciudad y se asocia a cualidades de apertura espacial, público, libertad y pluralismo. La morfología de la ciudad se invierte y las secciones del espacio privado cerrado forman redes de comunicación y de la vida cotidiana urbana reuniendo cualquier versión de actuación arquitectónica de lo construido y lo sin construir, mientras que el espacio público configura paisajes de abandono y olvido en la periferia de la ciudad, despojado de sus signos sociales conflictivos, símbolos vacíos y límites de los valores.

Así, en contraste con la ciudad moderna donde la vida urbana se desarrollaba en el espacio libre y el regreso a la normalidad se limitaba al islote destacando la protección de los ciudadanos, la terapia de los otros o la necesidad de clasificación para el bienestar de todos, en el paisaje de la ciudad posmoderna los lugares se transmutan y la vida urbana se sitúa en las zonas de exclusividad que ahora adquiere un signo positivo, mientras que lo público se identifica con la negatividad. Se trata de "la inversión del modelo de ciudad foucaultiano”, la cual se registra en otras áreas de la vida urbana de los estratos superiores (Paraskevópoulos 2008). En este enfrentamiento, en cuanto a las gated communities, la función específica de los 
límites, la limitación material del vecindario, es crucial. En el espacio urbano formado por los barrios cerrados, la condición de exclusiones y exclusividades se concentra en el concepto de distinción espacio-social: con todas las diferencias y escaladas funcionales y formales, la función de los límites de las gated communities en el espacio se traduce a veces como una prohibición $\mathrm{y}$, a veces, más moderado como el acceso controlado, pero siempre distingue entre "ellos" y los "otros", siempre excluyendo a "ellos" frente a los "otros" (Pantelidou 2019).

La generalización de la estructura limitada de la ciudad tiene como resultado el fortalecimiento del espacio privado y el debilitamiento al mismo tiempo del espacio público. El espacio urbano elimina sus cualidades público-políticas, se desurbaniza y degenera en un lugar de simple convivencia paralela. El espacio público, lo que queda de las áreas restringidas, debilitado por su estatus social, tiende a reducirse en un espacio físico de un analógicamente ya reducido individuo. En la medida de las apariencias y consolidación en la formación urbana contemporánea del fenómeno de barrios cerrados y otros puntos de control de acceso restringido exclusivo, destaca una nueva forma de organización, construcción y edificación del espacio urbano, una puerta urbanística que divide la ciudad por dentro y por fuera de las vallas. Realmente, lo que ilumina la perspectiva de las puertas urbanísticas es una ciudad de discriminación espacio-social y la intensidad de la brecha social, en la que el significado de vivienda se encuentra en la materialidad de la puerta de entrada y la vivienda se identifica como una condición urbana de aislamiento, privatización y cerramiento.

La intervención humana en el espacio - desde sus formas más primitivas, en el sentido de soberanía territorial como presencia única y apropiación del espacio natural, hasta las formas más avanzadas de arquitectura y tecnología espacial- siempre conlleva el significado de protección frente a cualquier tipo de amenaza que está fuera de sus límites (Dripps 1997). A una distancia de cada reducción de lo social en la naturaleza, en la modernidad occidental la búsqueda de la protección se entrelazó con la demarcación de control de espacio y accesos con temas de propiedad y poder (Rifkin 2001). Las gated communities son una versión de esta evolución, ya que se configura específicamente según los términos del tratado moderno.

En respuesta a la planificación urbana y las discriminaciones que intensifica, la arquitectura actual debe buscar formas de contribuir a los espacios y prácticas justas en lo que se refiere a los términos de las condiciones de vida de las personas en las ciudades. Una dirección para esto es la recuperación de su límite problemático y la investigación de un ethos espacial de justicia (Pantelidou 2020). En este contexto, la arquitectura debe estar a la altura de la amplitud de su carácter social y humanitario, pero también confrontar los límites de su dinámica. En cualquier caso, la relación entre arquitectura y derecho no se limita al diseño de cárceles y juzgados, sino que se extiende a reflexiones sobre las formas en que las ciudades que construimos expresan el concepto de derecho (Simon, Temple y Tobe 2013).

Diferentes pensadores entre ellos como Bakhtin (1984/1968) y Foucault (1982) nos enseñaron que la realidad cuando se crea a nivel institucional y estructural, otro tanto se crea en los regímenes de la práctica durante la conducta vivencial y participativa de la vida cotidiana. 
Ahora, la necesidad de resistir el control de las puertas urbanísticas de las gated communities plantea la cuestión de activar la arquitectura juntos en el campo de la crítica y en la escala de la vida cotidiana, estimulando una poética judicial de la arquitectura, para explorar formas en las que la arquitectura, en lo que a ella res-

\section{REFERENCIAS}

Boyle, Thomas Coraghessan. 1996. The Tortilla Curtain. Toronto: Penguin.

Bakhtin, Mikhail. 1984 [1968]. Rabelais and his World, trans. by H. Iswolsky. Bloomington: Indiana University Press.

Bauman, Zygmunt. 1997. Postmodernity and its discontents. New York: New York University Press.

Blakely, Edward y Snyder, Mary Gail. 1997. "Divided We Fall. Gated Communities in the United States". Architecture of Fear, editado por Nan Ellin and Edward Blakely. New York, NY: Princeton Architectural Press: 85-99.

Butler, Judith. 1997. The Psychic Life of Power: Theories in Subjection. Redwood City CA: Stanford University Press.

Debord, Guy. 2002 [1970]. Society of the Spectacle. St Petersburg FL: Black and Red.

Dripps, Robert Dunning. 1997. The first house: Myth, paradigm, and the task of architecture. London and Cambridge, MA: MIT Press. pecta podría contribuir a la creación de ciudades y relaciones justas. En la perspectiva de un ethos espacial de derecho, debemos reflexionar sobre la dinámica de los límites en el espacio y orientarnos a espacialidades de apertura y convivencia, abriendo las ciudades a la justa, común experiencia de las personas.

Foucault, Michel. 1982. "The Subject and Power". Critical Inquiry, 8, 4: 777-795.

Foucault, Michel. 1991. "Governmentality." The Foucault Effect: Studies in Governmentality, editado por Graham Burchell, Colin Gordon y Peter Miller. Chicago: University of Chicago Press: 87-104.

Grant, Jill y Mittelsteadt, Lindsey. 2004. “Types of gated communities". Environment and Planning B: Planning and Design. 31, 6: 913-30.

Harvey, David. 1992. "Social justice, Postmodernism and the City." International Journal of Urban and Regional Research, 16, 4: 588-601.

Kondylis, Panagiotis. 1991. Der Niedergang der burgerlichen Denk- und Lebensform. Die liberale Moderne und die massendemokratische Postmoderne. Weinheim: Acta Humaniora.

Lefas, Pavlos. 2009. Dwelling and Architecture: From Heidegger to Koolhaas. Berlin: JOVIS. 
Le Goix, Ronald. 2006. "Gated Communities as Predators of Public Resources: The Outcomes of Fading Boundaries between Private Management and Public Authorities in Southern California". Private Cities. Global and Local Perspectives, editado por Georg Glasze, Chris Webster and Klaus Frantz. London; New York: Routledge: 76-91.

Low, Setha. 2004. Behind the Gates: Life, Security, and the Pursuit of Happiness in Fortress America. London, Routledge.

Marcuse, Peter. 1995. "Not Chaos, but Walls: Postmodernism and the Partitioned City". Postmodern Cities and Spaces, editado por Sofia Watson and Kathie Gibson. Oxford: Blackwell: 243-53.

McKenzie, Evan. 2006. “The Dynamics of Privatopia: Private Residential Governance in the USA". Private Cities. Global and Local Perspectives, editado por Georg Glasze, Chris Webster and Klaus Frantz. London; New York: Routledge: 9-30.

Pantelidou, Charikleia. 2019. Gated Communities and Spatial-Social Exclusion: Form and Meaning Transformations in the Contemporary Urban Space. Athens: Nissos (in Greek).

Pantelidou, Charikleia. 2020. "The Spatial Ethos of the Carnivalesque: Urban Space as 'theprivate-within-the public". The City and Complexity: Life, Design and Commerce in the Built Environment, Proceedings of the AMPS -Architecture, Media,
Politics and Society- 2020 International Conference. University of London. Vol. 2: 180-187.

Paraskevopoulos, Nikos. 2008. "The extension of criminal law: real and symbolic dimensions". Honorary Volume for Anna Benaki-Psarouda: Criminal Sciences, Theory and Praxis. Athens: Sakkoula: 485496 (in Greek).

Petropoulou Chryssanthi. 2020. "Ciudades invisibles y cambio de habitus: narrativas cartográficas, poéticas y rebeldes. Ejemplos de Ciudad Bolívar (Bogotá) y Comuna 13 "San Javier (Medellín)". Narrativas, Geografias, Cartografiaspara viver, é preciso espaço e tempo, editado por Nelson Rego y Salete Kozel, en colaboración con Ana Francisca de Azevedo. Universidade do Minho, UFPR, UFRGS. Porto Alegre: Editora Compasso Lugar Cultura e Editora IGEO, Vol. 2: 669-723.

Rifkin, Jeremy. 2001. The Age of Access. New York: TarcherPerigee.

Sassen, Saskia. 2010. "Foreword: Urban Gating One Instance of a Larger Development?" Gated Communities: Social Sustainability in Contemporary and Historical Gated Developments, editado por Samer Bagaeen and Ola Uduku. London: Routledge.

Simon Jonathan, Temple Nicolas y Tobe Renée. 2013. Architecture and Justice: Judicial Meanings in the Public Realm. Surrey: Ashgate. 
Tsoukala, Kyriaki. 2010. "Introduction:

Postmodern aspects”. Postmodern aspects, editado por Kyriaki Tsoukala, Maria Daniil and Charikleia Pantelidou. Thessaloniki Epikendro: 15-50 (in Greek).

Webster, Chris. 2002. "Property Rights and THE Public Realm: Gates, Green Belts, and Gemeinschaft". Environment and Planning B: Planning and Design, 29: 397-412.
Wilson-Doenges, Georjeanna. 2000. “An exploration of sense of community and fear of crime in gated communities". Environment and Behavior 32, 5: 597-611.

Žižek, Slavoi. 1989. The Sublime Object of Ideology. Brooklyn, NY: Verso.

Žižek, Slavoi. 2001. Did Somebody Say Totalitarianism? Brooklyn, NY: Verso.
Charikleia Pantelidou es titulada de Arquitectura y Ciencias Sociales y doctora por el Departamento de Arquitectura de la Universidad Aristóteles de Tesalónica. Su trabajo de investigación tiene relación con enfoques interdisciplinarios del espacio y la Arquitectura. Su tesis doctoral estudia el fenómeno de los barrios cerrados en la ciudad moderna, además, ha elaborado investigación postdoctoral, becada por el Comité de Investigación de la Universidad Aristóteles de Tesalónica y la Fundación Nacional de Becas sobre la relación entre el espacio público y privado y la residencia colectiva moderna. Ha publicado artículos en revistas internacionales, libros colectivos, actas de congresos además de editar volúmenes colectivos en Grecia y en Inglaterra. Es autora del libro Comunidades cerradas y exclusión espacial-social: Transformaciones de forma y significado en el espacio urbano moderno, 2019, publicaciones Nisos. Enseña en la International Hellenic University. 


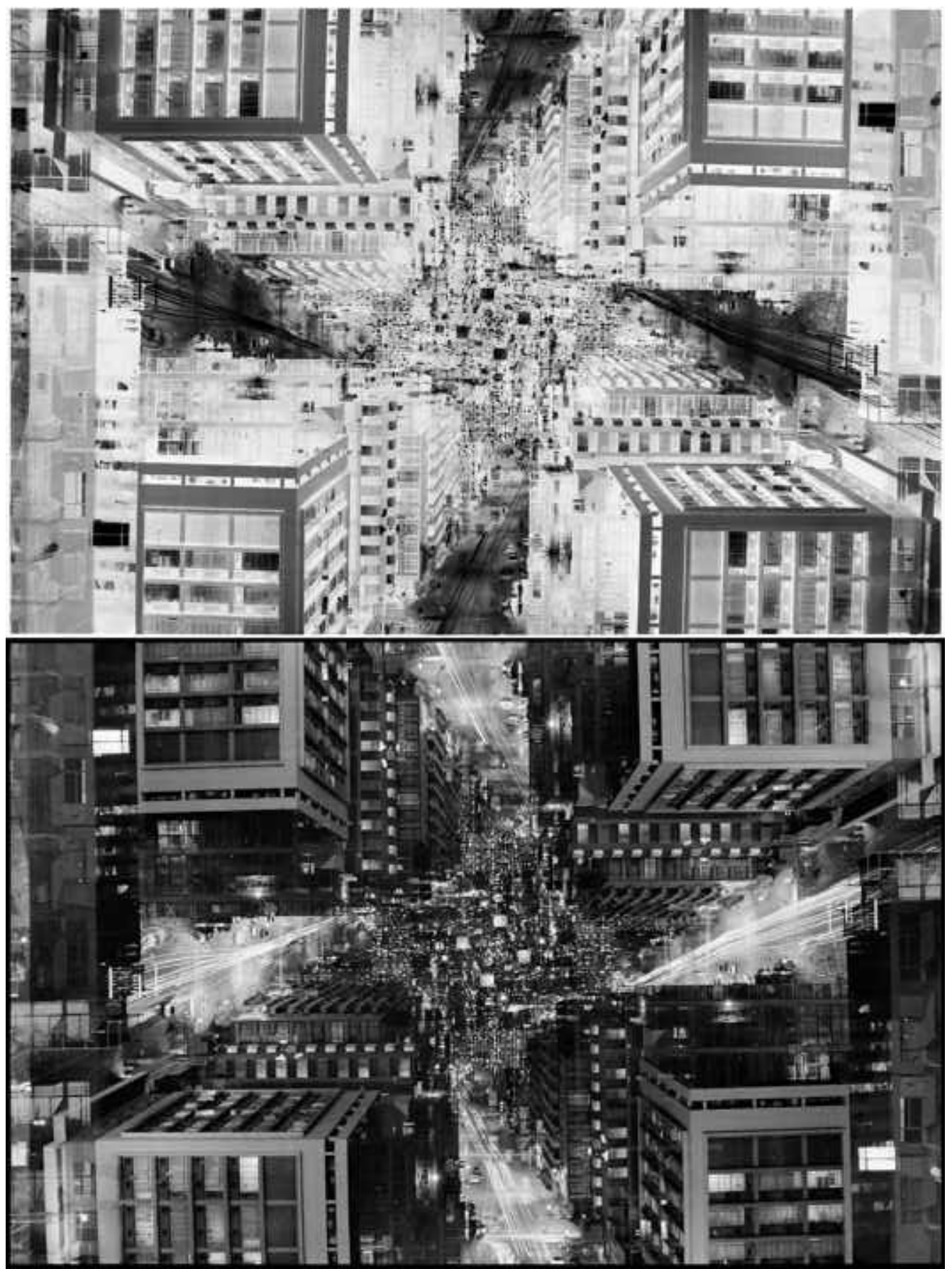

